\title{
Rational topological complexity
}

\author{
BARRY JESSUP \\ ANICETO MuRILLO \\ Paul-EugÈne Parent
}

\begin{abstract}
We give a new upper bound for Farber's topological complexity for rational spaces in terms of Sullivan models. We use it to determine the topological complexity in some new cases, and to prove a Ganea-type formula in these and other cases.
\end{abstract}

55M30, 55P62

\section{Introduction}

Motion planning is a rapidly growing area of research in

[14]. From the topological point of view, a motion planning algorithm has as input two states of the motion, ie, two arbitrary points in the configuration space $X$. As output it has to provide a continuous path between the two chosen states. In other words, such an algorithm consists of a section (not necessarily continuous) $s: X \times X \longrightarrow X^{I}$ of the unpointed path fibration $p: X^{I} \longrightarrow X \times X, \quad p(\sigma)=(\sigma(0), \sigma(1))$, where $X^{I}$ denotes the space of free paths on $X$.

One sees immediately that a section of $p$ can be chosen to be continuous if and only if the configuration space $X$ is contractible, and, indeed, any such contraction readily produces a continuous motion planning algorithm. Inspired by the work of Smale [18], Farber [2] took this observation as his starting point for defining the topological complexity of $X$, which is the following measure of the difficulty of finding a motion planning algorithm for a given configuration space:

Definition 1.1 [2] Let $X$ be a path-connected topological space. The topological complexity of $X, \operatorname{TC}(X)$, is the least integer $k$ for which there exists a covering of $X \times X$ formed by $k+1$ open sets $U_{1}, \ldots, U_{k+1}$, admitting continuous local sections $s_{i}: U_{i} \rightarrow X^{I}$ of $p$, ie, $p \circ s_{i}=1_{U_{i}}$, for each $i=1, \ldots, k+1$.

This is the sectional category (refer to Cornea, Lupton, Oprea and Tanré [1] and Schwarz [17]) of the path fibration. (Note that our definition differs from that of [2] by 
one, so that here, the topological complexity of a point is zero.) For a compendium of basic facts and known results on this homotopy invariant we refer to the excellent survey [3]. There, Farber poses in Section 31 the open problem of an "algebraic description of the rational version of $\mathrm{TC}(X)$ in terms of the Sullivan minimal model of $X ”$.

The first progress in this direction was the work of L Fernández, P Ghienne, T Kahl and L Vandembroucq. In [9] they introduced, for any simply connected space $X$, a lower bound for $\operatorname{TC}\left(X_{\mathbb{Q}}\right)$, namely $\operatorname{MTC}(X)$, which is an invariant of the rational homotopy type of $X$, and is defined in terms of a Sullivan model of $X$.

Later on, using the general approach of Fassò Velenik [4] for describing the sectional category of the rationalization of a given fibration, an algebraic description of $\operatorname{TC}\left(X_{\mathbb{Q}}\right)$ in terms of the Sullivan model of $X$ was explicitly presented by Lechuga and the second author in [16]. However, like that of [9], this description is not easy to handle.

Here, we give a simple upper bound for $\operatorname{TC}\left(X_{\mathbb{Q}}\right)$ and $\operatorname{MTC}(X)$ [9], which was inspired directly by the highly successful algebraic characterization of the LusternikSchnirelmann category of $X_{\mathbb{Q}}$ in terms of Sullivan minimal models given by Félix and Halperin in [5].

In the following, we will assume that our spaces are of the homotopy type of simply connected CW-complexes of finite type.

Let $(\Lambda V, d)$ (or simply $\Lambda V$ ) be a Sullivan model of $X$ and let $K \subset \Lambda V \otimes \Lambda V$ be the kernel of the multiplication $\mu: \Lambda V \otimes \Lambda V \rightarrow \Lambda V$. For any $n \geq 1$, denote by $K^{n}$ the $n$-th power of $K$, ie, the ideal generated by products of elements of $K$ of length at least $n$.

Definition 1.2 Consider the projection

$$
\Lambda V \otimes \Lambda V \stackrel{p_{m}}{\longrightarrow} \Lambda V \otimes \Lambda V / K^{m+1}
$$

Then,

(i) $\operatorname{tc}(X)$ is the smallest $m$ for which $p_{m}$ has a homotopy retraction as algebras

(ii) $\operatorname{mtc}(X)$ is the smallest $m$ for which $p_{m}$ has a homotopy retraction as $(\Lambda V \otimes \Lambda V)$-modules. 
Recall that, in this context, having a homotopy retraction means that in any Sullivan model of the projection,

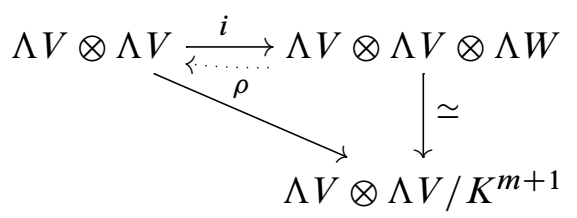

the map $i$ has a retraction $\rho: \Lambda V \otimes \Lambda V \otimes \Lambda W \rightarrow \Lambda V \otimes \Lambda V$, which is a map of a differential algebras, or $(\Lambda V \otimes \Lambda V)$-modules.

We prove:

Proposition 1.3 For any simply connected space $X$,

$$
\operatorname{MTC}(X) \leq \operatorname{TC}\left(X_{\mathbb{Q}}\right) \leq \operatorname{tc}(X) \quad \text { and } \quad \operatorname{MTC}(X) \leq \operatorname{mtc}(X) \leq \operatorname{tc}(X) .
$$

This simple upper bound is particularly interesting since it is an NP-hard problem to determine whether the topological complexity of a rational space is finite, given its minimal model as codification. Indeed, due to the well known inequality [2]

$$
\operatorname{cat}(X) \leq \mathrm{TC}(X) \leq 2 \operatorname{cat}(X),
$$

the finiteness of the topological complexity of a given space is equivalent to that of its LS-category. But, since determining the finiteness of the LS-category of a rational space is NP-hard (see Lechuga and Murillo [15, Theorem 2]), this shows that determining whether the topological complexity is finite is also NP-hard.

We find a class of spaces whose topological complexity attain this lower bound.

Theorem 1.4 Let $X$ be a space for which $\pi_{*}(X) \otimes \mathbb{Q}$ is finite dimensional and concentrated in odd degrees. Then,

$$
\operatorname{TC}\left(X_{\mathbb{Q}}\right)=\operatorname{dim} \pi_{*}(X) \otimes \mathbb{Q}=\operatorname{cat}\left(X_{\mathbb{Q}}\right) .
$$

Indeed, the last equality is a well known identity by Félix and Halperin [5] or Félix, Halperin and Thomas [7, Example 6, Section 29]. Here, the lower bound of the inequality $\operatorname{cat}(X) \leq \mathrm{TC}(X) \leq \operatorname{cat}(X \times X)$ of [2] is attained. Another consequence of Proposition 1.3 is a sharpening of [9, Proposition 6.2]. Recall that given $A$ a CDGA, its nilpotence index, denoted by nil $A$, is the least integer $n$ (possibly infinite) for which $A^{n+1}=0$. 
Proposition 1.5 Let $N$ be a CDGA of the rational homotopy type of the space $X$ with multiplication $\mu_{N}$. Then,

$$
\operatorname{TC}\left(X_{\mathbb{Q}}\right) \leq \operatorname{tc}(X) \leq \operatorname{nil} \operatorname{ker} \mu_{N} .
$$

Recall that the Ganea conjecture for LS-category stated that the LS-category of a space increases by one when taking a product with a sphere [10]. This conjecture was proved to be false in the general case by Iwase [12] but it remains true in the rational category. For example, see Félix, Halperin and Lemaire [6], Hess [11] and Jessup [13]. Here, a final application to the Ganea conjecture for rational topological complexity is obtained using mtc.

It is well known [2] that topological complexity satisfies the subadditive formula

$$
\mathrm{TC}(X \times Y) \leq \mathrm{TC}(X)+\mathrm{TC}(Y) .
$$

To the authors' knowledge, no example of strict inequality in the above has previously appeared in the literature. However, if $X=\mathbf{S}^{2} \cup_{f} e^{3}$ and $Y=\mathbf{S}^{2} \cup_{g} e^{3}$, where $f$ and $g$ are maps of degree 2 and 3 respectively, then in Section 3 we show that strict inequality does occur in this case. If we endow a given robot with configuration space $X$ with an extra articulated arm with $n$ degrees of freedom, the configuration space of the new robot is $X \times \mathbf{S}^{n}$, which satisfies

$$
\mathrm{TC}\left(X \times \mathbf{S}^{n}\right) \leq \mathrm{TC}(X)+\operatorname{TC}\left(\mathbf{S}^{n}\right)= \begin{cases}\mathrm{TC}(X)+1 & \text { if } n \text { is odd } \\ \mathrm{TC}(X)+2 & \text { if } n \text { is even }\end{cases}
$$

We do not know if equality holds in (1) for rational spaces. However we are able to show that equality does hold for mtc in all cases, and in some for tc, MTC and TC.

Theorem 1.6 If $X$ is a simply connected $C W$-complex of finite type and $n \geq 2$, then

$$
\operatorname{MTC}\left(X \times \mathbf{S}^{n}\right) \geq \operatorname{MTC}(X)+\operatorname{MTC}\left(\mathbf{S}^{n}\right)=\operatorname{MTC}(X)+\operatorname{TC}\left(\mathbf{S}^{n}\right) .
$$

Moreover,

$$
\operatorname{mtc}\left(X \times \mathbf{S}^{n}\right)=\operatorname{mtc}(X)+\operatorname{mtc}\left(\mathbf{S}^{n}\right)=\operatorname{mtc}(X)+\operatorname{MTC}\left(\mathbf{S}^{n}\right)=\operatorname{mtc}(X)+\operatorname{TC}\left(\mathbf{S}^{n}\right) .
$$

Though the following result can be proven directly from the characterization of TC given in the next section, here we obtain it as a corollary of Theorem 1.6.

Corollary 1.7 If $X$ is a formal, simply connected, rational $C W$-complex of finite type, and $n \geq 2$, then

$$
\operatorname{TC}\left(X \times \mathbf{S}^{n}\right)=\operatorname{TC}(X)+\operatorname{TC}\left(\mathbf{S}^{n}\right) .
$$


Acknowledgements The authors are grateful to José G Carrasquel and Yves Félix for their generous help with previous versions of this article. Our thanks also go to Daniel Tanré for pointing out to us the use of the generalized polyhedral product in describing the fat wedge. We also thank the referee for helpful remarks and suggestions. The research of the second author was supported by the Ministerio de Educación y Ciencia (grant MTM2010-18089), and the Junta de Andalucía (grants FQM-213 and P07-FQM-2863). The research of the first and third authors was partly supported by NSERC (Discovery Grants 45985 and 210206, respectively).

\section{Rational Topological Complexity}

We shall be using known results in rational homotopy for which the excellent reference Felix, Halperin and Thomas [7] is now standard. Here we present a summary of some basic facts. For any simply connected CW-complex of finite type $X$ (all spaces considered here shall be of this kind), its rationalization $X_{\mathbb{Q}}$ is a rational space (ie, its homotopy groups are rational vector spaces), together with a map $X \rightarrow X_{\mathbb{Q}}$ inducing isomorphisms in rational homotopy. On the other hand, to any space $X$ there corresponds, in a contravariant way, a Sullivan algebra, ie, a commutative differential graded algebra $(\Lambda V, d)$, called the minimal model of $X$, which is unique up to isomorphism and algebraically models the rational homotopy type of the space $X$, or equivalently, the homotopy type of its rationalization $X_{\mathbb{Q}}$. By $\Lambda V$ we mean the free commutative algebra generated by the graded vector space $V$, ie, $\Lambda V=T V / I$ where $T V$ denotes the tensor algebra over $V$ and $I$ is the ideal generated by $v \otimes w-(-1)^{|w||v|} w \otimes v$ for all $v, w \in V$, homogeneous elements of degrees $|v|$ and $|w|$ respectively. Moreover, as all spaces considered here are 1-connected, the differential $d$ satisfies the following minimality condition: for any element of $v \in V, d v$ is a polynomial in $\Lambda V$ with no linear term. This is known in this context as the nilpotence condition [7, page 138]. This correspondence yields an equivalence between the homotopy categories of 1-connected rational spaces of finite type and that of 1-connected rational commutative differential graded algebras (CDGA) of finite type.

We next give an algebraic description of $\operatorname{TC}\left(X_{\mathbb{Q}}\right)$, different from that of Lechuga and the second author [16], which is based on characterizations of the sectional category of a rational fibration given in terms of generalized fat wedges [4]. The fat wedge can also be seen as a generalized polyhedral product. If we use the diagonal map $\Delta: X \rightarrow X \times X$ to regard $(X \times X, X)$ as a CW pair, then by Félix and Tanré [8, Theorem 1] the $m$-th fat wedge is $\underline{(X \times X, X))^{\mathbf{S}^{m}}}$. 
Let $(\Lambda V, d)$ be a Sullivan model of $X$. The multiplication map of $\Lambda V$,

$$
\mu:(\Lambda V, d) \otimes(\Lambda V, d) \rightarrow(\Lambda V, d)
$$

is a model of the diagonal $\Delta: X \rightarrow X \times X$. Let $A$ denote $(\Lambda V, d) \otimes(\Lambda V, d)$ and $K \subset A$ the kernel of $\mu$. Then, a model of the $m$-th fat wedge associated to the diagonal $\Delta: X \rightarrow X \times X$ is given by the projection

$$
A^{\otimes^{m+1}} \stackrel{P}{\longrightarrow} A^{\otimes^{m+1}} / K^{\otimes^{m+1}} .
$$

If $A^{\otimes^{m+1}} \stackrel{M}{\longrightarrow} A$ denotes the iterated multiplication $\alpha_{1} \otimes \cdots \otimes \alpha_{m+1} \mapsto \alpha_{1} \cdots \alpha_{m+1}$ and

$$
A^{\otimes^{m+1}} \stackrel{j}{\hookrightarrow} A^{\otimes^{m+1}} \otimes \Lambda W \stackrel{\simeq}{\longrightarrow} A^{\otimes^{m+1}} / K^{\otimes^{m+1}}
$$

is a relative Sullivan model of $P$, then we have the following proposition:

Proposition 2.1 $\quad$ (a) [4, Proposition 8.4.1] $\mathrm{TC}\left(X_{\mathbb{Q}}\right)$ is the least $m$ for which there is a map of differential graded algebras

$$
\rho: A^{\otimes^{m+1}} \otimes \Lambda W \rightarrow A
$$

with $\rho j=M$.

(b) $[9$, Section 6] $\operatorname{MTC}(X)$ is the least $m$ for which there is a map of differential graded $A$-modules $\rho: A^{\otimes^{m+1}} \otimes \Lambda W \rightarrow A$ with $\rho j=M$.

Henceforth, we use (a) and (b) above as definitions of TC and MTC.

The proof of Proposition 1.3 is now immediate:

Proof of Proposition 1.3 With the notation of the above, simply note that the multiplication map $M$ above takes $K^{\otimes^{m+1}}$ to $K^{m+1}$, and so induces a map of differential graded algebras

$$
A^{\otimes^{m+1}} / K^{\otimes^{m+1}} \stackrel{\widetilde{M}}{\longrightarrow} A / K^{m+1} .
$$

Thus, any homotopy retraction of $A \rightarrow A / K^{m+1}$ will, essentially by precomposition with $\widetilde{M}$, induce the desired map $\rho$.

From this, some important consequences are deduced. The first, Proposition 1.5 (see Section 1) is a sharpening of [9, Proposition 6.2]. 
Proof of Proposition 1.5 Let $\varphi:(\Lambda V, d) \stackrel{\simeq}{\longrightarrow} N$ be the minimal model of $N$ (and hence of $X)$ and denote by $\mu$ the multiplication in $\Lambda V$. As $(\varphi \otimes \varphi)(\operatorname{ker} \mu) \subset \operatorname{ker} \mu_{N}$ we have, for any $m$, a commutative square:

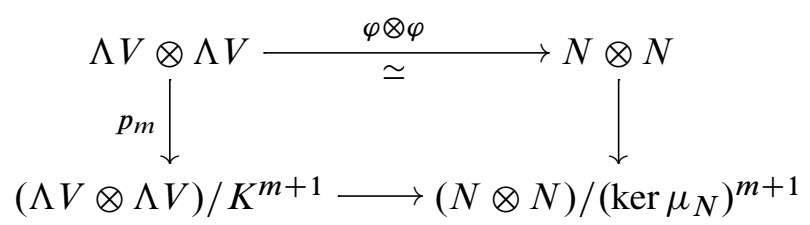

Whenever $m \geq$ nil $\operatorname{ker} \mu_{N},\left(\operatorname{ker} \mu_{N}\right)^{m+1}=0$ and so this becomes the triangle:

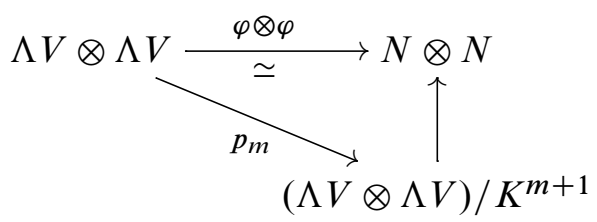

This readily implies the existence of a homotopy retraction of $p_{m}$, and so

$$
\operatorname{TC}(X) \leq \operatorname{tc}(X) \leq \text { nil } \operatorname{ker} \mu_{N} .
$$

Recall that if

$$
\cup_{\mathbb{K}}: H^{*}(X ; \mathbb{K}) \otimes H^{*}(X ; \mathbb{K}) \rightarrow H^{*}(X ; \mathbb{K})
$$

denotes the cup product for any field $\mathbb{K}$, the main cohomological lower bound for topological complexity is given by [2, Theorem 7]

$$
\mathrm{TC}(X) \geq \operatorname{nil} \operatorname{ker} \cup_{\mathbb{K}} .
$$

From Proposition 1.5 we can thus immediately obtain the main result of [16]. Recall that a simply connected space $X$ is said to be formal if its rational homotopy type depends only on its rational cohomology algebra.

Corollary 2.2 For any formal space $X$,

$$
\operatorname{TC}\left(X_{\mathbb{Q}}\right)=\operatorname{nil} \operatorname{ker} \cup_{\mathbb{Q}} .
$$

Another class of spaces for which rational topological complexity can easily be computed via Proposition 1.3 is given by Theorem 1.4. We begin its proof with the following observation:

Lemma 2.3 If $\mu: \Lambda V \otimes \Lambda V \rightarrow \Lambda V$ is the multiplication map, then ker $\mu$ is generated, as an ideal, by $\{v \otimes 1-1 \otimes v \mid v \in V\}$. 
Proof Let $I=\langle\{v \otimes 1-1 \otimes v \mid v \in V\}\rangle$. It is clear that $I \subset \operatorname{ker} \mu$, and that moreover, $(\Lambda V \otimes \Lambda V) / I \cong \Lambda V$ via the homomorphism induced by $\mu$.

We now proceed to the proof of Theorem 1.4:

Proof of Theorem 1.4 Let $(\Lambda V, d)$ be a minimal model of $X$, and $\left\{v_{1}, \ldots, v_{n}\right\}$ a basis of $V$ such that $\left|v_{i}\right| \leq\left|v_{i+1}\right|$. If

$$
U=\operatorname{ker} \mu \cap \Lambda^{1}(V \oplus V)=\operatorname{span}\left\{v_{i} \otimes 1-1 \otimes v_{i} \mid 1 \leq i \leq n\right\},
$$

then $\operatorname{ker} \mu: \Lambda V \otimes \Lambda V \rightarrow \Lambda V$ is just $\Lambda V \otimes \Lambda^{+} U$, and $K^{m}=(\operatorname{ker} \mu)^{m}=\Lambda V \otimes \Lambda^{\geq m} U$. Since $\pi_{*}(X) \otimes \mathbb{Q} \cong U=U^{\text {odd }}$ and $\operatorname{dim} U=n, K^{n+1}=0$, and so, by Proposition 1.3, $\operatorname{TC}\left(X_{\mathbb{Q}}\right) \leq \operatorname{tc}(X) \leq n$.

We conclude by noting that $\operatorname{TC}\left(X_{\mathbb{Q}}\right) \geq \operatorname{cat}\left(X_{\mathbb{Q}}\right)=\operatorname{dim} V=n$.

\section{The Ganea conjecture for rational topological complexity}

We begin here with the example of strict inequality of the subadditive formula on products for TC mentioned in the introduction. The following may be compared to Farber [3, Theorem 19.1].

Lemma 3.1 Suppose $X$ and $Y$ are well-pointed. If $X \vee Y \hookrightarrow X \times Y$ is a homotopy equivalence, then

$$
\mathrm{TC}(X \vee Y) \leq \max \{\mathrm{TC}(X), \operatorname{TC}(Y)\} .
$$

Proof If we regard $X \vee Y$ as the subset $X \times\left\{y_{0}\right\} \cup\left\{x_{0}\right\} \times Y$ of the product $X \times Y$, then $(X \vee Y) \times(X \vee Y)$ is the union of the following subsets of $X \times Y \times X \times Y$ :

$$
\begin{aligned}
(X \vee Y) \times(X \vee Y)=X \times\left\{y_{0}\right\} \times X \times\left\{y_{0}\right\} \cup X \times\left\{y_{0}\right\} \times\left\{x_{0}\right\} \times Y \\
\cup\left\{x_{0}\right\} \times Y \times X \times\left\{y_{0}\right\} \cup\left\{x_{0}\right\} \times Y \times\left\{x_{0}\right\} \times Y .
\end{aligned}
$$

On the other hand, since our spaces are well pointed, the inclusion $X \vee Y \hookrightarrow X \times Y$ is a cofibration, and thus, as it is a homotopy equivalence, it is also a deformation retract (though not necessarily strong); see Spanier [19, Corollary 10, page 31]. Hence, we use the deformation retraction of $X \times Y$ onto $X \times\left\{y_{0}\right\} \cup\left\{x_{0}\right\} \times Y$ to deduce that

$$
\begin{aligned}
& X \times\left\{y_{0}\right\} \times\left\{x_{0}\right\} \times\left\{y_{0}\right\} \cup\left\{x_{0}\right\} \times\left\{y_{0}\right\} \times\left\{x_{0}\right\} \times Y, \\
& \left\{x_{0}\right\} \times\left\{y_{0}\right\} \times X \times\left\{y_{0}\right\} \cup\left\{x_{0}\right\} \times Y \times\left\{x_{0}\right\} \times\left\{y_{0}\right\}
\end{aligned}
$$


are also deformation retracts of

$$
\begin{aligned}
& X \times\left\{y_{0}\right\} \times\left\{x_{0}\right\} \times Y, \\
& \left\{x_{0}\right\} \times Y \times X \times\left\{y_{0}\right\},
\end{aligned}
$$

respectively. Therefore,

$$
(X \times X) \vee(Y \times Y)=X \times\left\{y_{0}\right\} \times X \times\left\{y_{0}\right\} \cup\left\{x_{0}\right\} \times Y \times\left\{x_{0}\right\} \times Y
$$

is a deformation retract of $(X \vee Y) \times(X \vee Y)$. We denote this retraction by $r$ :

$$
(X \times X) \vee(Y \times Y) \stackrel{j}{\stackrel{j}{\rightleftarrows}}(X \vee Y) \times(X \vee Y)
$$

Now, consider homotopy sections of the fibrations $X^{I} \rightarrow X \times X$ and $Y^{I} \rightarrow Y \times Y$ over coverings $\left\{U_{1}, \ldots, U_{n}\right\}$ and $\left\{V_{1}, \ldots, V_{m}\right\}$ of $X \times X$ and $Y \times Y$ respectively. Assume $n \geq m$. Using the homotopy lifting property, we may assume those sections to be base point preserving whenever any of the elements of these coverings contain the base point. Thus, there are homotopy sections of

$$
(X \vee Y)^{I} \longrightarrow(X \vee Y) \times(X \vee Y) \stackrel{r}{\longrightarrow}(X \times X) \vee(Y \times Y)
$$

over the covering $\left\{U_{1} \vee V_{1}, \ldots, U_{m} \vee V_{m}, U_{m+1}, \ldots, U_{n}\right\}$. Finally, consider the induced homotopy sections of $(X \vee Y)^{I} \longrightarrow(X \vee Y) \times(X \vee Y)$ over the covering

$$
\left\{r^{-1}\left(U_{1} \vee V_{1}\right), \ldots, r^{-1}\left(U_{m} \vee V_{m}\right), r^{-1}\left(U_{m+1}\right), \ldots, r^{-1}\left(U_{n}\right)\right\} .
$$

If $X=\mathbf{S}^{2} \cup_{f} e^{3}$ and $Y=\mathbf{S}^{2} \cup_{g} e^{3}$, where $f$ and $g$ are maps of degree 2 and 3 respectively, then $\mathrm{TC}(X)$ and $\mathrm{TC}(Y)$ are positive, and $X \vee Y \hookrightarrow X \times Y$ is a homotopy equivalence. Then, by all of the above, this is also a deformation retract and, by Lemma 3.1, we have

$$
\mathrm{TC}(X \times Y)=\mathrm{TC}(X \vee Y) \leq \max \{\mathrm{TC}(X), \operatorname{TC}(Y)\}<\mathrm{TC}(X)+\mathrm{TC}(Y) .
$$

As stated in the introduction, it is open whether, for a rational simply connected CWcomplex X of finite type, one always has the equality $\operatorname{TC}\left(X \times \mathbf{S}^{n}\right)=\operatorname{TC}(X)+\operatorname{TC}\left(\mathbf{S}^{n}\right)$. However, this Ganea formula does hold for mtc, as we prove in our final result, Theorem 1.6. Note that, for $\mathbf{S}^{n}$,

$$
\operatorname{MTC}\left(\mathbf{S}^{n}\right)=\operatorname{mtc}\left(\mathbf{S}^{n}\right)=\operatorname{TC}\left(\mathbf{S}_{\mathbb{Q}}^{n}\right)=\operatorname{tc}\left(\mathbf{S}^{n}\right)=\operatorname{TC}\left(\mathbf{S}^{n}\right)= \begin{cases}1 & \text { if } n \text { is odd } \\ 2 & \text { if } n \text { is even. }\end{cases}
$$

The first three equalities trivially hold as $\mathbf{S}^{n}$ is a formal space while the fourth is well known [3]. For the second note that $\operatorname{TC}\left(\mathbf{S}_{\mathbb{Q}}^{n}\right)=\operatorname{nil} \operatorname{ker} \cup_{\mathbb{Q}}=\operatorname{TC}\left(\mathbf{S}^{n}\right)$. 
Proof of Theorem 1.6 We first prove subadditivity of mtc, ie,

$$
\operatorname{mtc}(X \times Y) \leq \operatorname{mtc}(X)+\operatorname{mtc}(Y) .
$$

Let $\Lambda V$ and $\Lambda W$ be Sullivan models of $X$ and $Y$. Write $V \oplus V=V^{2}$ and observe that, if $K_{V}$ denotes the kernel of the multiplication $\Lambda V^{2} \rightarrow \Lambda V$, then $K_{V \oplus W}$ is generated as a $\Lambda(V \oplus W)$-module by $\{v \otimes 1-1 \otimes v, w \otimes 1-1 \otimes w \mid v \in V, w \in W\}$ (see Lemma 2.3). Thus, for $m, n \geq 1$, there is a natural morphism of algebras

$$
\Lambda\left(V^{2} \oplus W^{2}\right) / K_{V \oplus W}^{m+n+1} \longrightarrow \Lambda\left(V^{2}\right) / K_{V}^{m+1} \otimes \Lambda\left(W^{2}\right) / K_{W}^{n+1}
$$

which induces, via the lifting lemma [7, Proposition 12.9], a morphism $h$ between the Sullivan models of the quotients:

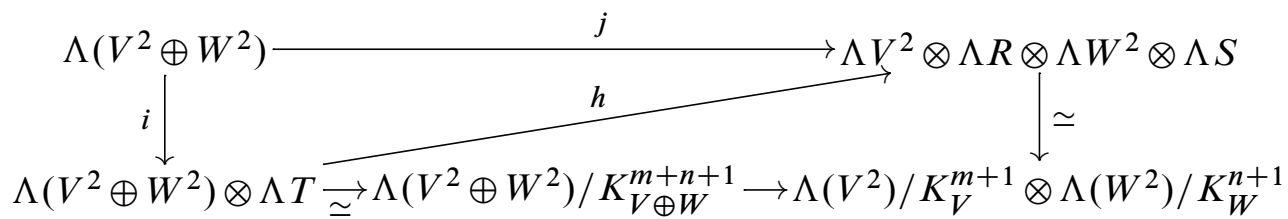

Thus, if $j$ has a retraction $\rho$ (either as a morphism of algebras or $\Lambda(V \oplus W)$-modules), then $\rho h$ is a retraction of $i$. This proves the assertion and also the subadditivity of "rational" TC.

We now prove the reverse inequality for mtc whenever $Y$ is a sphere. Again, let $(\Lambda V, d)$ be a Sullivan model of $X$ and $\left(\Lambda U_{n}, d\right)$ denote the model of an $n$-sphere $\mathbf{S}^{n}$, so that $U_{2 k+1}=\operatorname{span}\{u\}, U_{2 k}=\operatorname{span}\{x, y\}, d u=0=d x, d y=x^{2},|u|$ is odd and $|x|$ is even. In what follows, we suppress the dependence on $n$ wherever possible.

Let $A=\Lambda V \otimes \Lambda V, C=\Lambda U_{n} \otimes \Lambda U_{n}$, and $B=A \otimes C$, all differentials being those from the products. Denote by $K$ and $L$ the kernel of the multiplication in $A$ and $B$ respectively. Consider in $C$ the element $z \otimes 1-1 \otimes z \in U_{n} \oplus U_{n}$, denoted by $z-z^{\prime}$ henceforth, and observe that $z-z^{\prime} \in L$.

Now define

$$
\gamma= \begin{cases}u-u^{\prime} & \text { if } n \text { is odd } \\ \left(x-x^{\prime}\right)^{2} & \text { if } n \text { is even. }\end{cases}
$$

Note that

$$
\gamma \in \begin{cases}L & \text { if } n \text { is odd, } \\ L^{2} & \text { if } n \text { is even, }\end{cases}
$$

and that, $[\gamma] \neq 0$ in $H^{*}(C)$, and thus it is also nonzero in $H^{*}(B)$. 
Hence, as graded differential vector spaces, we may write

$$
C=\langle\gamma\rangle \oplus M
$$

and define a map $p: C \rightarrow \mathbb{Q}$ of graded differential vector spaces by $p(k \gamma+m)=k$. Note that $p$ is homogenous of degree $-|\gamma|$.

This allows us to write $B=(A \otimes\langle\gamma\rangle) \oplus(A \otimes M)$, the direct sum being now one of differential $A$-modules. Now define two maps of differential $A$-modules $\sigma: A \rightarrow B$ and $\tau: B \rightarrow A$ by

$$
\sigma(\alpha)=\alpha \gamma \quad \text { and } \quad \tau\left(\alpha_{0} \gamma+\alpha_{1} m\right)=\alpha_{0} .
$$

It is easy to check that, for all $m, \sigma\left(K^{m}\right) \subset L^{m+\operatorname{mtc}\left(\mathbf{S}^{n}\right)}$, which implies that the following diagram commutes:

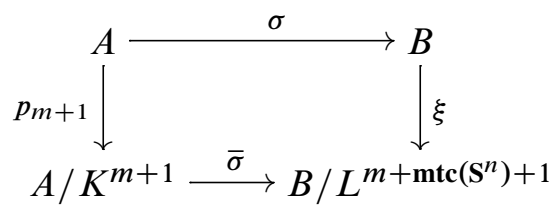

Moreover, $\tau$ is a retraction of $\sigma$. Thus, if $\xi$ has a homotopy retraction, $p_{m+1}$ does as well. This proves that

$$
\operatorname{mtc}(X)+\operatorname{mtc}\left(\mathbf{S}^{n}\right) \leq \operatorname{mtc}\left(X \times \mathbf{S}^{n}\right),
$$

and so establishes the Ganea formula for mtc.

The inequality $\operatorname{MTC}\left(X \times \mathbf{S}^{n}\right) \geq \operatorname{MTC}(X)+\operatorname{TC}\left(\mathbf{S}^{n}\right)$ is established in a similar fashion, which we now outline. We use the same notation as before.

Briefly, the map

$$
\bar{\sigma}: A^{\otimes^{m+1}} \longrightarrow B^{\otimes^{m+\mathrm{MTC}\left(\mathbf{S}^{n}\right)+1}}
$$

defined by

$$
\alpha_{1} \otimes \cdots \otimes \alpha_{m+1} \mapsto \begin{cases}\alpha_{1} \otimes \cdots \otimes \alpha_{m+1} \otimes\left(u-u^{\prime}\right) & n \text { odd, } \\ \alpha_{1} \otimes \cdots \otimes \alpha_{m+1} \otimes\left(x-x^{\prime}\right) \otimes\left(x-x^{\prime}\right) & n \text { even, }\end{cases}
$$

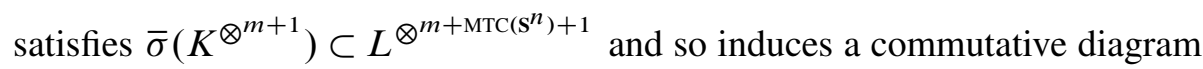

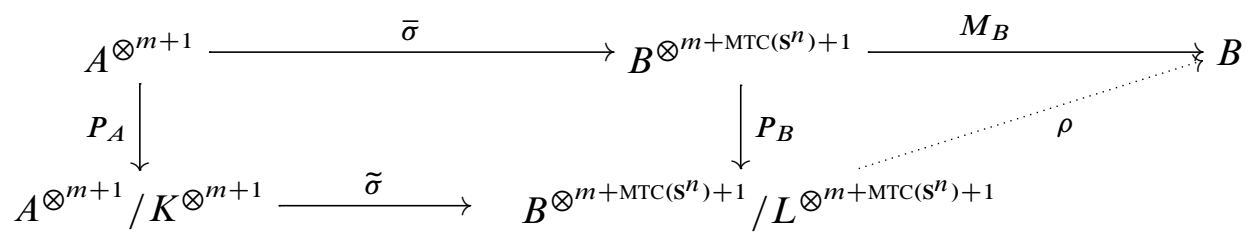


where $M_{B}$ is multiplication. Thus, if there is (up to a relative Sullivan model) a map $\rho$ as shown with $\rho P_{B} \simeq M_{B}$, postcomposition with $\tau$ defined earlier shows that

$$
\operatorname{MTC}(X) \leq \operatorname{MTC}\left(X \times \mathbf{S}^{n}\right)-\operatorname{MTC}\left(\mathbf{S}^{n}\right) .
$$

We end with the following:

Conjecture For all spaces $X$ with the homotopy type of simply connected CWcomplexes of finite type,

$$
\operatorname{TC}\left(X_{\mathbb{Q}}\right)=\operatorname{tc}(X) .
$$

\section{References}

[1] O Cornea, G Lupton, J Oprea, D Tanré, Lusternik-Schnirelmann category, Math. Surveys and Monographs 103, Amer. Math. Soc. (2003) MR1990857

[2] M Farber, Topological complexity of motion planning, Discrete Comput. Geom. 29 (2003) 211-221 MR1957228

[3] M Farber, Topology of robot motion planning, from: "Morse theoretic methods in nonlinear analysis and in symplectic topology", (P Biran, O Cornea, F Lalonde, editors), NATO Sci. Ser. II Math. Phys. Chem. 217, Springer, Dordrecht (2006) 185-230 MR2276952

[4] A Fassò Velenik, Relative homotopy invariants of the type of the Lusternik-Schnirelmann category, $\mathrm{PhD}$ thesis, Freien Universität Berlin (2002) Available at http://www.diss.fu-berlin.de/diss/receive/ FUDISS_thesis_000000001150

[5] Y Félix, S Halperin, Rational LS category and its applications, Trans. Amer. Math. Soc. 273 (1982) 1-38 MR664027

[6] Y Félix, S Halperin, J-M Lemaire, The rational LS category of products and of Poincaré duality complexes, Topology 37 (1998) 749-756 MR1607732

[7] Y Félix, S Halperin, J-C Thomas, Rational homotopy theory, Grad. Texts in Math. 205, Springer, New York (2001) MR1802847

[8] Y Félix, D Tanré, Rational homotopy of the polyhedral product functor, Proc. Amer. Math. Soc. 137 (2009) 891-898 MR2457428

[9] L Fernández Suárez, P Ghienne, T Kahl, L Vandembroucq, Joins of DGA modules and sectional category, Algebr. Geom. Topol. 6 (2006) 119-144 MR2199456

[10] T Ganea, Some problems on numerical homotopy invariants, from: "Symposium on Algebraic Topology (Battelle Seattle Res. Center, Seattle WA, 1971)", (P J Hilton, editor), Lecture Notes in Math. 249, Springer, Berlin (1971) 13-22 MR0339147 
[11] K P Hess, A proof of Ganea's conjecture for rational spaces, Topology 30 (1991) 205-214 MR1098914

[12] N Iwase, Ganea's conjecture on Lusternik-Schnirelmann category, Bull. London Math. Soc. 30 (1998) 623-634 MR1642747

[13] B Jessup, Rational approximations to L-S category and a conjecture of Ganea, Trans. Amer. Math. Soc. 317 (1990) 655-660 MR956033

[14] J-C Latombe, Robot motion planning, Kluwer intl series in engineering and comp. sci.: Robotics, Kluwer Acad. Publ., Dordrecht (1990)

[15] L Lechuga, A Murillo, Complexity in rational homotopy, Topology 39 (2000) 89-94 MR1710993

[16] L Lechuga, A Murillo, Topological complexity of formal spaces, from: "Topology and robotics", (M Farber, R Ghrist, M Burger, D Koditschek, editors), Contemp. Math. 438, Amer. Math. Soc. (2007) 105-114 MR2359032

[17] A S Schwarz, The genus of a fiber space, Dokl. Akad. Nauk SSSR (N.S.) 119 (1958) 219-222 MR0102812

[18] S Smale, On the topology of algorithms, I, J. Complexity 3 (1987) 81-89 MR907191

[19] E H Spanier, Algebraic topology, Springer, New York (1981) MR666554

BJ, P-EP: Department of Mathematics and Statistics, University of Ottawa 585 King Edward Ave, Ottawa K1N6N5, Canada

AM: Departmento de Algebra Geometría y Topología, University of Malaga Ap 59, 29080 Malaga, Spain

Barry.Jessup@uottawa.ca, aniceto@uma.es, pparent@uottawa.ca

Received: 22 March $2012 \quad$ Revised: 11 April 2012 